\title{
Comparing inductive and deductive analysis techniques to understand health service implementation problems: a case study of childhood vaccination barriers
}

Carissa Bonner ${ }^{1 *}$ D, Jane Tuckerman², Jessica Kaufman ${ }^{2}$, Daniel Costa ${ }^{3,4}$, David N. Durrheim ${ }^{5}$, Lyndal Trevena ${ }^{1}$, Susan Thomas ${ }^{5}$ and Margie Danchin ${ }^{2,6,7}$

\begin{abstract}
Background: Effective implementation requires a comprehensive understanding of individual, organisational and system determinants. This study aimed to compare inductive and deductive analysis techniques to understand a complex implementation issue. We used childhood vaccination as a case study, an issue with wide-ranging barriers contributing to low-vaccine uptake internationally.

Methods: The study is based on the Behaviour Change Wheel framework, which was derived from several levels of theory: the 3 components of the COM-B framework (capability, opportunity and motivation) can be mapped to the 14 domains of the Theoretical Domains Framework (TDF), which is based on 84 underlying constructs. We first conducted a review of systematic reviews of parent-level barriers to childhood vaccination. Subsequently we (1) inductively coded these barriers into a data-driven framework, using thematic analysis, and (2) deductively mapped the barriers to COM-B and TDF domains and constructs. These processes were undertaken by two authors independently, and discrepancies were resolved through discussion. Inductive and deductive results were compared.

Results: The inductive process coded 583 descriptions of barriers identified from the literature into a framework of 74 barriers in 7 categories. The initial definitions used to map the barriers to deductive domains/constructs led to 89\% agreement at the domain level. Resolving discrepancies required further definitions at the construct level. Of the 14 TDF domains, 10 were clearly identified in the data from the barrier reviews. Some domains were not specific enough to differentiate between types of barriers (e.g. Environmental Context and Resources), while other domains were not represented in the review data (e.g. Behavioural Regulation).
\end{abstract}

\footnotetext{
* Correspondence: carissa.bonner@sydney.edu.au

${ }^{1}$ Faculty of Medicine and Health, School of Public Health, The University of Sydney, Sydney, Australia

Full list of author information is available at the end of the article
}

(c) The Author(s). 2021 Open Access This article is licensed under a Creative Commons Attribution 4.0 International License, which permits use, sharing, adaptation, distribution and reproduction in any medium or format, as long as you give appropriate credit to the original author(s) and the source, provide a link to the Creative Commons licence, and indicate if changes were made. The images or other third party material in this article are included in the article's Creative Commons licence, unless indicated otherwise in a credit line to the material. If material is not included in the article's Creative Commons licence and your intended use is not permitted by statutory regulation or exceeds the permitted use, you will need to obtain permission directly from the copyright holder. To view a copy of this licence, visit http://creativecommons.org/licenses/by/4.0/. The Creative Commons Public Domain Dedication waiver (http://creativecommons.org/publicdomain/zero/1.0/) applies to the data made available in this article, unless otherwise stated in a credit line to the data. 
Conclusions: Using both inductive and deductive analysis techniques can help achieve a more comprehensive understanding of barriers to health service implementation. The inductive categories represented the review data in a clearer way than the theoretical domains, with better differentiation; but the missing deductive domains were useful as a way to identify additional issues to investigate further. Both analysis techniques resulted in a comprehensive list of barriers to vaccination that would not have been achieved using either approach alone. We recommend a hybrid approach combining TDF with broader frameworks, for future researchers conducting evidence syntheses.

Keywords: TDF, COM-B, Implementation, Childhood vaccination

\section{Contributions to the literature}

- Deductive theoretical analysis techniques to understand implementation problems, such as the TDF and COM-B, may raise different issues compared to inductive data-driven analysis techniques

- This paper describes a process for comparing inductive and deductive analysis techniques to understand an implementation challenge of global significance

- We describe an analysis process using several levels of framework development (84 constructs underlying the 14 TDF domains, which link to the 3 COM-B components) and identify new directions to improve the specificity of theoretical behavioural constructs in future research

- The paper illustrates how inductive and deductive analysis techniques synergise to produce a more comprehensive understanding of health service barriers than using either approach alone

\section{Background}

Effective implementation of a health service programme, guideline or treatment requires understanding a wide range of system, organisational and individual determinants of uptake [1]. This may involve reviewing existing literature for well-established problems or conducting original research if the issue is new. Incorporating theoretical frameworks can ensure all possible drivers are considered [2].

The use of theoretical frameworks enables an understanding of the mechanisms of change from individual to system levels, which can then be targeted in interventions. Multiple theories are used in healthcare, from simple models of individual health behaviour change like the Theory of Planned Behaviour [3], to broader systems thinking approaches to map the complexity of policy drivers [4]. The Behaviour Change Wheel (BCW) is one approach that attempts to bring individual and system level factors together [5], based on the COM-B (capability, opportunity, motivation-behaviour) framework that synthesises 14 behavioural constructs in the Theoretical Domains Framework (TDF) [6] into broader categories.
The TDF summarises the many overlapping constructs in the behaviour change literature and was developed through expert consensus from 128 theoretical constructs in 33 theoretical models of behaviour [7]. It provides an overview of 14 key theoretical constructs that explain health behaviour and is a descriptive framework rather than a theory of causality. A separate systematic review of 19 frameworks for behaviour change interventions led to the $\mathrm{BCW}$, which aims to guide the development of interventions by connecting the determinants of behaviour with behaviour change techniques [5]. Developed in conjunction with the $\mathrm{BCW}$, and at its central core, is the COM-B framework which proposes that behaviour is a product of the interaction between capability (psychological or physical), opportunity (social or physical) and motivation (automatic or reflective) [5, 7].

The COM-B and TDF have been mapped to each other, but there is some duplication of the current 14 TDF domains across the COM-B components. Table 1 summarises this theoretical relationship.

Primary research is often used to identify barriers to implementation in different health service contexts, and this is the approach generally used with the TDF [7]. Some issues have been well researched, but this evidence must be synthesised in order to inform comprehensive intervention design [8]. Previous reviews have applied theoretical frameworks to help with this. For example, the $\mathrm{BCW}$ can be used to describe interventions in terms of broader functions [9], and the COM-B can be used to display barriers and facilitators at multiple levels (patient, provider, system) [9]. The TDF can be used together with the COM-B to group barriers and facilitators of health outcomes [10], or as a stand alone framework [11].

A deductive analysis technique using theory-driven constructs may identify different implementation issues compared to inductive techniques that are data-driven. A deductive application of theory ensures that all psychological constructs relevant to behaviour are considered, even if research has not identified every construct. However, since these theoretical frameworks are based heavily on psychological theory, the internal 'motivation' aspect is more clearly defined than the more external 'opportunity' aspect. This imbalance does not necessarily 
Table 1 Relationships between the TDF and COM-B (adapted from Tables 2 and 3 in Cane et al.) [6]

\begin{tabular}{|c|c|}
\hline COM-B components & TDF domain definitions \\
\hline \multirow[t]{2}{*}{ Capability: Psychological } & Knowledge: An awareness of the existence of something \\
\hline & Behavioural Regulation: Anything aimed at managing or changing objectively observed or measured actions \\
\hline $\begin{array}{l}\text { Capability: Psychological } \\
\text { and physical }\end{array}$ & Skills: An ability or proficiency acquired through practice \\
\hline Capability: Physical & $\begin{array}{l}\text { Memory, Attention and Decision Processes: The ability to retain information, focus selectively on aspects of } \\
\text { the environment and choose between two or more alternatives }\end{array}$ \\
\hline Opportunity: Physical & $\begin{array}{l}\text { Environmental Context and Resources: Any circumstance of a person's situation or environment that } \\
\text { discourages or encourages the development of skills and abilities, independence, social competence and } \\
\text { adaptive behaviour }\end{array}$ \\
\hline Opportunity: Social & $\begin{array}{l}\text { Social influences: Those interpersonal processes that can cause individuals to change their thoughts, feelings } \\
\text { or behaviours }\end{array}$ \\
\hline \multirow[t]{4}{*}{ Motivation: Reflective } & $\begin{array}{l}\text { Beliefs about Consequences: Acceptance of the truth, reality or validity about outcomes of a behaviour in a } \\
\text { given situation }\end{array}$ \\
\hline & $\begin{array}{l}\text { Beliefs about Capabilities: Acceptance of the truth, reality or validity about an ability, talent or facility that a } \\
\text { person can put to constructive use }\end{array}$ \\
\hline & Intentions: A conscious decision to perform a behaviour or a resolve to act in a certain way \\
\hline & Goals: Mental representations of outcomes or end states that an individual wants to achieve \\
\hline \multirow[t]{2}{*}{$\begin{array}{l}\text { Motivation: Reflective } \\
\text { and automatic }\end{array}$} & $\begin{array}{l}\text { Social/Professional Role and Identity: A coherent set of behaviours and displayed personal qualities of an } \\
\text { individual in a social or work setting }\end{array}$ \\
\hline & Optimism: The confidence that things will happen for the best or that desired goals will be attained \\
\hline \multirow[t]{2}{*}{ Motivation: Automatic } & $\begin{array}{l}\text { Reinforcement: Increasing the probability of a response by arranging a dependent relationship, or contingency, } \\
\text { between the response and a given stimulus }\end{array}$ \\
\hline & $\begin{array}{l}\text { Emotion: A complex reaction pattern, involving experiential, behavioural, and physiological elements, by which } \\
\text { the individual attempts to deal with a personally significant matter or event }\end{array}$ \\
\hline
\end{tabular}

align with the prevalence and significance of practical issues in health service implementation, which might be defined as 'physical opportunity'. A hybrid approach can be used to address this $[12,13]$, but the extra time and expertise required need to be weighed against the potential benefits.

The aim of this paper is to compare inductive and deductive analysis techniques applied to the same implementation issue and illustrate how these processes can complement each other. We use parent uptake of childhood vaccination as an example of an international issue with wide ranging barriers identified in multiple reviews.

\section{Method}

\section{Theoretical approach}

The study was based on the BCW framework because it incorporates both individual and system level barriers to behaviour and is based on several levels of theory: the 3 components of the COM-B framework can be mapped to the 14 domains of the TDF, which is based on 84 underlying constructs [5].

\section{Context: The Vaccine Barriers Assessment Tool (VBAT) project}

This analysis is based on data gathered for the Vaccine Barriers Assessment Tool (VBAT) project, which aims to design and validate a survey tool to diagnose the causes of under-vaccination in children under 5 years. Developed in Australia and New Zealand, VBAT aims to incorporate both access and acceptance barriers in a comprehensive tool which will include both short and long form versions, for different uses. An overview of systematic reviews of primary studies on barriers to childhood vaccination was conducted, and 583 descriptions of parental barriers to childhood vaccination uptake were extracted and inductively grouped into categories [14]. Barriers were extracted if they were reported from or relevant to the specific perspective of parents of children under 5 years; barriers from the perspective of health professionals or the health system alone were not included. The findings of the review were thematically organised into a framework of barriers. In a separate deductive process, the 583 barrier descriptions were mapped to the 14 domain version of the TDF, to check whether any theoretical determinants of childhood vaccine uptake were missing in the systematic review data. The purpose of this exercise for the VBAT project was to ensure that a comprehensive pool of potential survey questions could be generated that captured both access and psychological or acceptance barriers. The inductive review and development of the VBAT items will be reported separately (manuscript in preparation [15]). In the results of this article, we describe the utility of using both inductive inductive and deductive analysis 
techniques to identifying drivers of vaccination. Specific terms are used as outlined in Table 2.

\section{Process}

Figure 1 illustrates the inductive and deductive processes, supported by regular group meetings with all authors to discuss each step. We used the perspective of parents (not health professionals or health systems), which affected the way the deductive categories were applied. The prevalence of domains was examined to determine missing theoretical constructs in the data.

\section{Results}

\section{Mapping inductive barriers to deductive domains}

The initial definitions used to compare inductive barriers with theoretical domains/constructs led to $89 \%$ agreement at the domain level. For example, we specified that all barriers relating to the clinic setting will be under the domain of Environmental Context and Resources. Resolving disagreements for the domains and subsequent constructs required further definitions at the construct level before $100 \%$ agreement was reached. Table 3 illustrates this for the domain of Environmental Context and Resources, where we decided that issues relating to how appointment times are managed will be under the construct of Organisational culture/climate, while issues relating to inconvenient access for the parent will be under the construct of Person $\mathrm{x}$ Environment Interaction. The full list of definitions in available in Appendix.

Figure 2 shows the number of barriers represented in each theoretical domain. Table 4 shows the relationship between deductive COM-B components and TDF domains, and inductive barriers identified in systematic reviews of primary research. Of the 14 TDF domains, 10 were definitively present in inductive data while 4 domains were not covered in the initial coding: Optimism, Intentions, Goals and Behavioural Regulation (with the exception of two very general barriers for Intentions and Goals with no further explanation). Two domains grouped many different concepts under generic terms (Beliefs within Beliefs about Consequences, Barriers and Facilitators within Environmental Context/Resources). Of the 84 constructs within the 14 TDF domains, many were not identified in the inductive data. This is shown in yellow in Appendix.

\section{Discussion}

Overall, we found it useful to synthesise health service implementation barriers using both inductive and deductive analysis techniques to gain a comprehensive understanding of the barriers to childhood vaccination. The inductive data-driven categories represented the primary research data in a clearer way than the deductive theoretical domains, with better differentiation; but the four missing theoretical domains were useful as a way to identify key gaps to be addressed in the item pool for developing a new tool to diagnose the causes of childhood under-vaccination.

Resolving conflicts at the domain level was relatively straighforward, with $100 \%$ agreement reached quickly. However, there were some barriers that could have been placed in several domains. For example, previous experience of vaccine side effects could be framed as knowledge, beliefs or salient events. Resolving conflicts at the construct level was more difficult because many constructs within a domain were very similar when applied to the brief barrier descriptions extracted from reviews, for example the influence of family member opinions could fit within group identity, social norm or social pressure. The decisions made at construct level were arguably more subjective than the domain level, but both needed to be considered to make sense of many barriers that could be framed in different ways.

For this study, it was necessary to go into more theoretical detail than the commonly used frameworks: the

Table 2 Term definitions and examples for inductive vs deductive analysis techniques

\begin{tabular}{|c|c|c|}
\hline $\begin{array}{l}\text { Level of } \\
\text { categorisation }\end{array}$ & Inductive terms & Deductive terms \\
\hline Low level (specific) & $\begin{array}{l}\text { Barrier descriptions refers to the } 583 \text { individual descriptions } \\
\text { of implementation issues extracted from systematic reviews } \\
\text { in the VBAT review of the vaccination uptake literature }\end{array}$ & $\begin{array}{l}\text { Construct refers to the more detailed list of } 84 \text { unique theoretical } \\
\text { concepts that informed the TDF and COM-B frameworks }\end{array}$ \\
\hline Example & Belief that the vaccine is more dangerous than the illness & Consequents \\
\hline Mid level & $\begin{array}{l}\text { Barrier refers to the } 74 \text { groups of barrier descriptions across } \\
\text { studies identified in the VBAT review of the vaccination } \\
\text { uptake literature }\end{array}$ & $\begin{array}{l}\text { Domain refers to the } 14 \text { broad categories of behavioural drivers } \\
\text { described in the most recent version of the TDF (Theoretical } \\
\text { Domains Framework) }\end{array}$ \\
\hline Example & Concern about vaccine safety & Beliefs about consequences \\
\hline High level (broad) & $\begin{array}{l}\text { Category refers to the } 7 \text { groups of barriers identified in the } \\
\text { VBAT review of the vaccination uptake literature }\end{array}$ & $\begin{array}{l}\text { Component refers to the } 6 \text { components in the simplest theory- } \\
\text { driven framework of behavioural drivers, the COM-B (Capability, } \\
\text { Opportunity, Motivation - Behaviour) }\end{array}$ \\
\hline Example & Concerns and beliefs & Reflective motivation \\
\hline
\end{tabular}


SYSTEMATIC REVIEW OF REVIEWS (led by JK, JT, MD)

Results: 583 identified barriers to vaccination

INDUCTIVE PROCESS (led by JK, JT)

Expertise: Cochrane methods, vaccination

Time taken: 2 weeks

- Step 1: JK conducted initial coding

- Step 2: JK \& JT discussed and agreed on preliminary thematic framework

- Step 3: JT conducted independent coding

- Step 4: JK \& JT discussed all discrepancies and refined final theme groupings

- Step 5: JK applied final groupings to data, which was checked by JT (74 barriers in 7 categories)
DEDUCTIVE PROCESS (led by CB, JT)

Expertise: behavioural models, vaccination

Time taken: 2 weeks

- Step 1: CB conducted initial coding

- Step 2: CB \& JT discussed and agreed on preliminary interpretation of theoretical domains/constructs

- Step 3: JT conducted independent coding

- Step 4: CB \& JT discussed all discrepancies and refined final interpretation of theoretical domains

- Step 5: CB applied final interpretation to data, which was checked by JT ( 84 constructs in 14 domains)

GROUP DISCUSSION TO COMPARE THE 2 PROCESSES (all authors)

Expertise: vaccination, public health, general practice, psychometric assessment,

behavioural science, science communication

Time taken: 1 day (in person workshop)

- Inductive process: individual ranking of the importance of barriers for each category,

discussed as a group for inclusion in VBAT item pool

- Deductive process: cross-tabulations to identify missing domains/constructs in deductive data considered for inclusion in VBAT item pool as a group

APPLICATION TO VBAT PROJECT (all authors)

Questionnaire item pool created based on final hybrid inductive/deductive barrier list

Cognitive testing of items to reduce item pool \& validation of questionnaire

Fig. 1 Inductive and deductive processes

COM-B and TDF. Importantly, the gaps identified in our inductive review would not have been found if the analysis had only been done at the COM-B level, as all six components were addressed by the 10 inductive barrier categories. In addition, the $14 \mathrm{TDF}$ domains were still not specific enough for two coders to reliably map the barrier data so we were required to go back a step to the 84 theoretical constructs that informed the TDF development. We found it helpful to use a combination of domain and construct level to map the data. A previous review using the TDF identified some issues that could not be mapped to the TDF, including clinician and patient characteristics. However, some of these could be mapped at the construct level depending on the framing, such as under professional identity, skills, environment $\mathrm{x}$ person and resources constructs [16].

Table 3 Example of definitions required to code TDF domains

\begin{tabular}{|c|c|c|}
\hline Domain & Construct & Notes on decision making \\
\hline \multirow{6}{*}{$\begin{array}{l}\text { Environmental Context and Resources (any circumstance of a person's } \\
\text { situation or environment that discourages or encourages the development } \\
\text { of skills and abilities, independence, social competence and adaptive behaviour) }\end{array}$} & Environmental stressors & Role of media \\
\hline & Resources/material resources & Cost issues, lack of supply \\
\hline & Organisational culture/climate & $\begin{array}{l}\text { How clinic is managed } \\
\text { (e.g. appointment time) }\end{array}$ \\
\hline & Salient events/critical incidents & $\begin{array}{l}\text { Specific adverse event/illness } \\
\text { in past }\end{array}$ \\
\hline & Person $\times$ Environment Interaction & $\begin{array}{l}\text { Inconvenience to specific } \\
\text { parent (e.g. location) }\end{array}$ \\
\hline & Barriers and facilitators & $\begin{array}{l}\text { General access factors/catch } \\
\text { all for 'other' }\end{array}$ \\
\hline
\end{tabular}

Note: there were 14 domains and 84 constructs. Coders CB and JT made notes on their decisions about how the construct was to be applied ot the data, with examples above and full notes in Appendix 


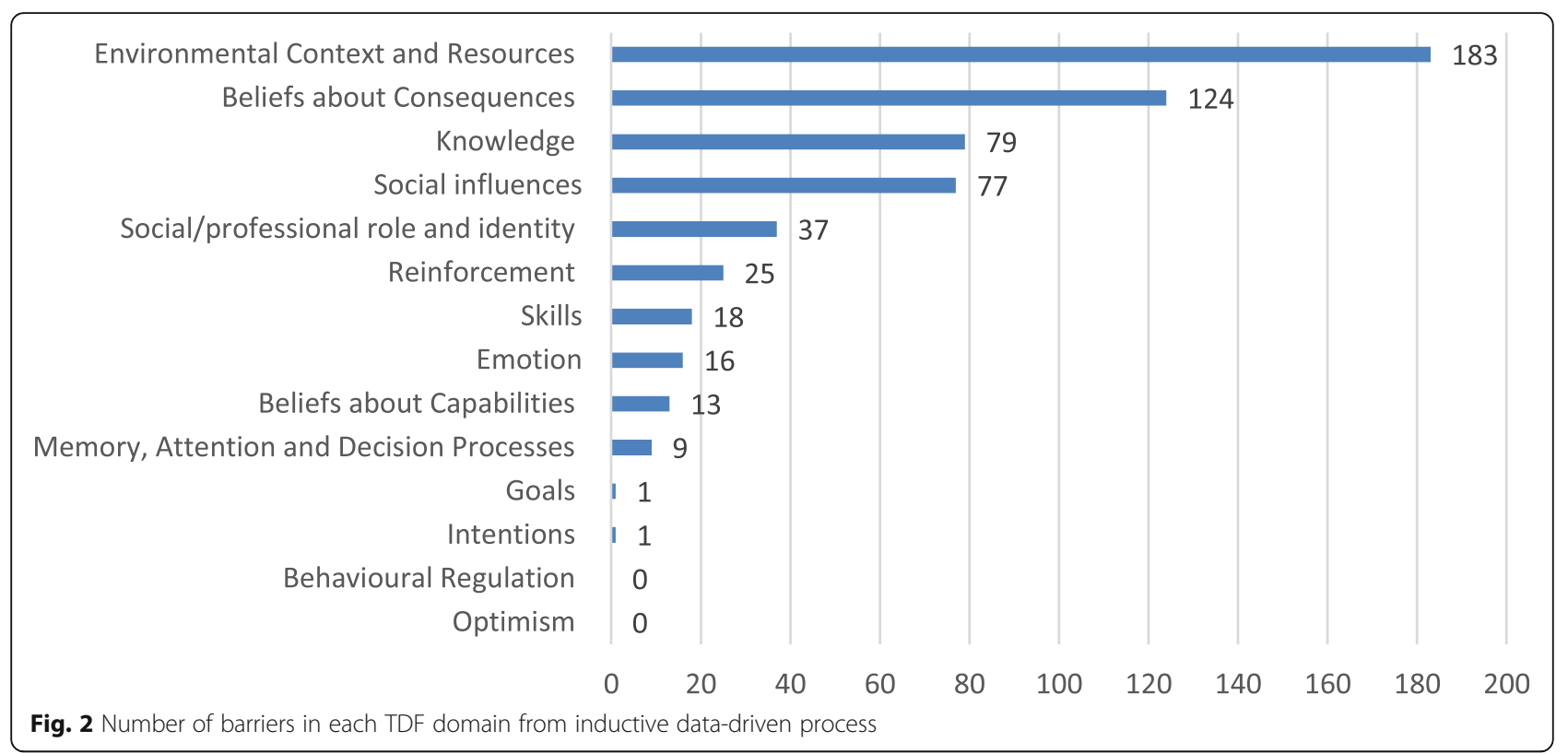

\section{Practical implications}

This paper provides analysis techniques for anyone seeking to understand an implementation issue that already has a large amount of qualitative and/or quantitative researchcomplementing an earlier paper that focuses on how to apply the TDF in primary qualitative research [7]. There are several practical implications for other researchers seeking to comprehensively understand implementation barriers using theoretical frameworks in this way. Firstly, researchers need to decide on very specific framing for a health situation. In our case, we decided we would only consider the parent perspective on vaccinating their child, which determined how we framed barriers relating to the doctors' knowledge. Conducting this process from the health professional perspective would produce different results in terms of the theoretical constructs identified in the literature. We included both barriers to the intervention and barriers to implementation but other projects may need to distinguish between these. Secondly, the COM-B framework was not specific enough with uneven

Table 4 Relationship between inductive and deductive concepts

\begin{tabular}{lll}
\hline COM-B component & Deductive concepts (TDF domains) & Inductive concepts (data-driven barriers) \\
\hline Capability & Knowledge & Lack of information about vaccination, false contraindications \\
& Skills & Staff are unpleasant or poor communication, language barriers \\
& Memory, Attention and Decision Processes & Reminder notice, missed opportunities, forgot \\
& *Behavioural Regulation & Not represented \\
Opportunity & Environmental Context and Resources & First child, low income, media, distance, supply, cost, time \\
& Social influences & Social exclusion, peer influence, trust, compliance, natural immunity \\
Motivation & Social/professional role and identity & Traditional beliefs and customs, role of parent, lack of coordinated care \\
& Beliefs about Capabilities & Can control pathogens child is exposed to, lower parental satisfaction with care \\
& $*$ Optimism & Not represented \\
& Beliefs about Consequences & Anticipated guilt, vaccine efficacy, disease severity/susceptibility, pain \\
& Reinforcement & Well-baby clinic counselling, benefit to others, vaccination delay at 3 months \\
& $*$ Intentions & Practices about health and prevention ( $n=1$ with lenient interpretation) \\
& $*$ Goals & Lack of motivation ( $n=1$ with lenient interpretation) \\
& Emotion & Anxiety about vaccination, fear of needles, psychosocial distress
\end{tabular}

*Note: These 4 domains were not included in the first round of coding. Intentions and goals were later included after discussion with a very lenient interpretation of the inductive barriers to maximise the number of domains covered, given the aim of the exercise was to generate questionnaire items covering all possible behavioural influences. No inductive barriers could be interpreted as behavioural regulation or optimism 
explanation of different barrier types, so researchers may need to go into more detail at domain and construct level to interpret the data. Thirdly, theory was useful for identifying gaps in an inductive review of literature, but inductive categories made more sense for the specific implementation topic. The value of using deductive theory-driven analysis techniques may depend on available resources, given this process took 2 authors with prior knowledge of behavioural frameworks around 2 weeks for coding and discussion. For our purposes, this review will inform the development of a diagnostic tool to measure the causes of undervaccination, requiring us to include the widest possible range of behavioural drivers. For other projects, it may be more prudent to focus only on the theoretical drivers that are within an organisation's control to address or to identify inductive issues from the perspective of key stakeholders to ensure their interest and support. Future questionnaire developers may benefit from reviewing existing validated survey items prior to a literature review, so that barriers can be linked to established items at the same time.

\section{Theoretical implications}

More generally, this study has implications for theoretical frameworks commonly used in implementation science. Some constructs are vague and became catch alls, such as barriers and facilitators. Others are too specific and hard to distinguish, particularly group vs social norms, which could be combined into one category. In our experience, the decision was often between constructs in different domains, rather than constructs within a domain, suggesting that there are some issues with the way the TDF domains are differentiated. On the other hand, the construct level was often too subjective and detailed to identify clear gaps in data. This suggests that overarching frameworks like the COM-B and TDF need to be supplemented with more contextspecific frameworks for different health areas (e.g. prevention versus treatment of infectious disease), targets of behaviour change (e.g. parents versus doctors), and the context (e.g. higher resource settings where psychological barriers may be more important, versus lower resource settings where practical access issues require greater differentiation). Another option would be to use broad implementation frameworks that include practical issues like cost, such as the Consolidated Framework for Implementation Research (CFIR) [17]. Other researchers have found it helpful to combine the TDF and CFIR for a more comprehensive approach [1]. A third option would be to add more specific domains to the next version of the TDF to better differentiate between issues relating to 'Environmental Context and Resources'. In our review, this covered a very wide range of issues: socio-economic issues such as having low income, societal issues like the influence of media, health system issues like vaccine supply and cost, and individual access issues like distance and time. This was found to be a catch all category in many previous reviews of clinicians and patients using the TDF $[16,18-22]$, so is not limited to the issue of vaccination barriers. For example, a review of barriers to low back pain guidelines found this domain was common to $4 / 5$ clinician behaviours while many other domains were not covered at all [20]. Another review on diabetic screening identified 17 barriers in this domain versus 6 for the next most common domain [18]. Further development of this construct may need to be specific to different health topics.

For the purpose of the VBAT study, we aimed to identify the widest possible range of behavioural barriers documented in the literature, not the relationships between them, so a framework approach was appropriate. We framed all concepts as 'barriers' by reversing concepts framed as facilitators where required, for consistency. VBAT will be used to identify the presence of key access and/or acceptance barriers in specific populations. Once identified, the key barriers would require more specific models or theories to guide intervention development, which may frame the same construct as either facilitator or barrier.

\section{Strengths and limitations}

This study involved independent coding for both inductive and deductive analysis techniques. Our team included a wide variety of expertise to help contextual framing for theoretical constructs as applied to inductive barriers. The limitations include restricting our review data to parent barriers only, which affected the way that health professionals' and heatlh system barriers were conceptualised. We also applied only one overarching framework based on behaviour change models and acknowledge that there are many other approaches to this theoretical issue.

In conclusion, using both inductive and deductive analysis techniques can help achieve a more comprehensive understanding of health service implementation problems, but the TDF approach needs to be refined in the context of vaccination. We recommend a hybrid approach combining TDF with frameworks such as CFIR, for future researchers conducting evidence syntheses using a theoretical approach. The process is subjective so requires a wide range of expertise to reduce biased interpretation and to maximise utility of the identified barriers for the specified purpose. 


\section{Appendix}

Table 5 Decision making notes by domain and construct (green/yellow $=$ not represented in data; orange $=$ only included after discussion; blue = 'catch all' domains that didn't differentiate well)

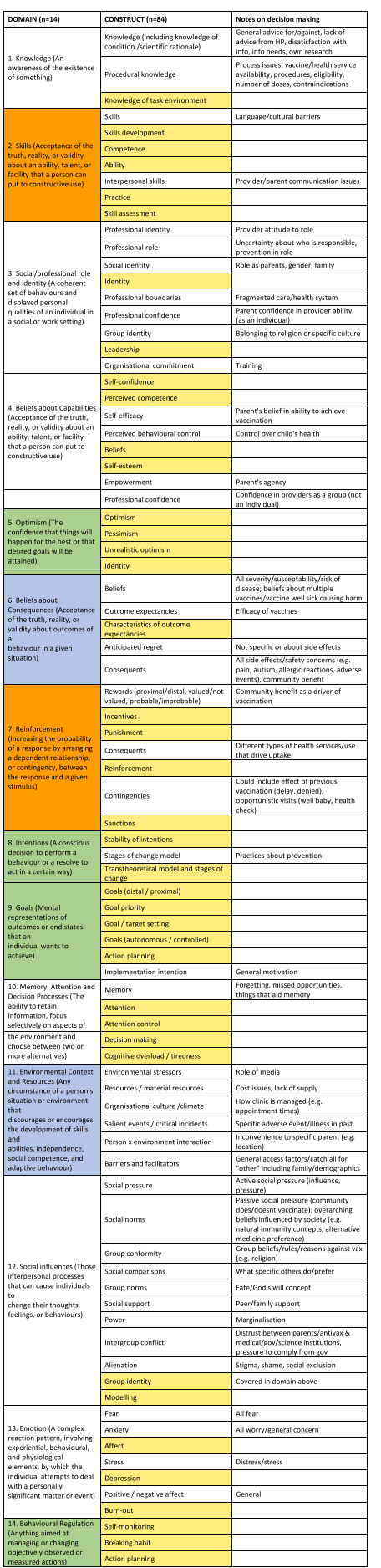

\section{Abbreviations}

BCW: Behaviour Change Wheel; TDF: Theoretical Domains Framework: COMB Model: Capability-Opportunity-Motivation-Behaviour Model; VBAT: Vaccine Barriers Assessment Tool; CFIR: Consolidated Framework for Implementation Research

\section{Acknowledgements}

We thank Michael Fajardo for assistance with searching for the original systematic review, and Carys Batcup for assistance finding other reviews that have used the COM-B and TDF frameworks and managing references.

\section{Authors' contributions}

CB conceived/designed the study, conducted the analysis, and drafted the paper. JT and JK conducted the analysis and were major contributors in writing the manuscript. DC, DD, LT, ST and MD contributed to group discussions to design the analysis approach and interpret the results and revised the manuscript. The authors read and approved the final manuscript and are accountable for the work.

\section{Authors' information}

Not applicable

\section{Funding}

The project was funded by a National Health and Medical Research Council grant from the Australian government (NHMRC Project Grant \#1164200).

\section{Availability of data and materials}

Data available on request from Carissa Bonner (carissa.bonner@sydney.edu. au).

\section{Declarations}

Ethical approval and consent to participate

Ethical approval was not required for this secondary analysis of published reviews.

\section{Consent for publication}

Not applicable

\section{Competing interests}

The authors declare that they have no comepting interests.

\section{Author details}

${ }^{1}$ Faculty of Medicine and Health, School of Public Health, The University of Sydney, Sydney, Australia. ${ }^{2}$ Vaccine Uptake Group, Murdoch Children's Research Institute, Melbourne, Australia. ${ }^{3}$ School of Psychology, The University of Sydney, Sydney, Australia. ${ }^{4}$ Pain Management Research Institute, Royal North Shore Hospital, Sydney, Australia. ${ }^{5}$ School of Medicine and Public Heatlh, University of Newcastle, Callaghan, Australia. ${ }^{6}$ Department of Paediatrics, University of Melbourne, Melbourne, Australia. ${ }^{7}$ Department of General Medicine, Royal Childrens Hospital, Melbourne, Australia.

Received: 14 March 2021 Accepted: 25 August 2021 Published online: 15 September 2021

\section{References}

1. Birken SA, Powell BJ, Presseau J, Kirk MA, Lorencatto F, Gould NJ, et al. Combined use of the Consolidated Framework for Implementation Research (CFIR) and the Theoretical Domains Framework (TDF): a systematic review. Implement Sci. 2017;12(1):2. https://doi.org/10.1186/s13012-0160534-z.

2. Michie S, Atkins L, West R. The Behaviour Change Wheel: A Guide to Designing Interventions. London: Silverback Publishing; 2014. www.beha viourchangewheel.com.

3. Sniehotta FF, Presseau J, Araújo-Soares V. Time to retire the theory of planned behaviour. Health Psychol Rev. 2014;8(1):1-7. https://doi.org/10.1 080/17437199.2013.869710.

4. McLeroy K. Thinking of systems. Am J Public Health. 2006;96(3):402. https:// doi.org/10.2105/AJPH.2005.084459. 
5. Michie S, van Stralen MM, West R. The behaviour change wheel: a new method for characterising and designing behaviour change interventions. Implement Sci. 2011;6(1):42. https://doi.org/10.1186/1748-5908-6-42.

6. Cane J, O'Connor D, Michie S. Validation of the theoretical domains framework for use in behaviour change and implementation research. Implement Sci. 2012;7(1):37. https://doi.org/10.1186/1748-5908-7-37.

7. Atkins L, Francis J, Islam R, O'Connor D, Patey A, Ivers N, et al. A guide to using the Theoretical Domains Framework of behaviour change to investigate implementation problems. Implement Sci. 2017;12(1):77. https:// doi.org/10.1186/s13012-017-0605-9.

8. Kastner M, Tricco AC, Soobiah C, Lillie E, Perrier L, Horsley T, et al. What is the most appropriate knowledge synthesis method to conduct a review? Protocol for a scoping review. BMC Med Res Methodol. 2012;12(1):114. https://doi.org/10.1186/1471-2288-12-114.

9. McDonagh LK, Saunders JM, Cassell J, et al. Application of the COM-B model to barriers and facilitators to chlamydia testing in general practice for young people and primary care practitioners: a systematic review. Implement Sci. 2018;13(1):1-19. https://doi.org/10.1186/s13012-018-0821-y.

10. Göstemeyer G, Baker SR, Schwendicke F. Barriers and facilitators for provision of oral health care in dependent older people: a systematic review. Clin Oral Investig. 2019;23(3):979-93. https://doi.org/10.1007/s00784019-02812-4

11. Craig LE, McInnes E, Taylor N, Grimley R, Cadilhac DA, Considine J, et al. Identifying the barriers and enablers for a triage, treatment, and transfer clinical intervention to manage acute stroke patients in the emergency department: a systematic review using the theoretical domains framework (TDF). Implement Sci. 2016;11(1):1-18. https://doi.org/10.1186/s13012-0160524-1.

12. Fereday J, Adelaide N, Australia S, Eimear M-CA. Demonstrating rigor using thematic analysis: a hybrid approach of inductive and deductive coding and theme development. Int J Qual Methods. 2006;5(1):80-92. https://doi. org/10.1177/160940690600500107.

13. Xu W, Zammit K. Applying thematic analysis to education: a hybrid approach to interpreting data in practitioner research. Int J Qual Methods. 2020;19:1-9. https://doi.org/10.1177/1609406920918810.

14. Kaufman J, Tuckerman J, Durrheim D, et al. Barriers to uptake of childhood vaccination: protocol for a review of systematic reviews; 2019. https://doi. org/10.26188/5DEF2F21A87CE.

15. Kaufman J, Tuckerman J, Bonner C, Durrheim D, Costa D, Trevena L, et al. Vaccine Barriers Assessment Tool: a global overview of childhood vaccine uptake barriers, Short Oral Presentation, 17th National Immunisation Conference. Perth: Public Health Association of Australia; 2021.

16. Thompson W, Tonkin-Crine S, Pavitt SH, McEachan RRC, Douglas GVA, Aggarwal VR, et al. Factors associated with antibiotic prescribing for adults with acute conditions: an umbrella review across primary care and a systematic review focusing on primary dental care. J Antimicrob Chemother. 2019;74(8):2139-52. https://doi.org/10.1093/jac/dkz152.

17. Damschroder L, Aron DC, Keith RE, Kirsh SR, Alexander JA, Lowery JC. Fostering implementation of health services research findings into practice: a consolidated framework for advancing implementation science. Implement Sci. 2009:4(1):50. https://doi.org/10.1186/1748-5908-4-50

18. Graham-Rowe E, Lorencatto F, Lawrenson JG, Burr JM, Grimshaw JM, Ivers $N M$, et al. Barriers to and enablers of diabetic retinopathy screening attendance: a systematic review of published and grey literature. Diabet Med. 2018;35(10):1308-19. https://doi.org/10.1111/dme.13686.

19. Atkins L, Sallis A, Chadborn T, Shaw K, Schneider A, Hopkins S, et al. Reducing catheter-associated urinary tract infections: a systematic review of barriers and facilitators and strategic behavioural analysis of interventions. Implement Sci. 2020;15(1):1-22. https://doi.org/10.1186/s13012-020-01001-2.

20. Hall AM, Scurrey SR, Pike AE, et al. Physician-reported barriers to using evidence-based recommendations for low back pain in clinical practice: a systematic review and synthesis of qualitative studies using the Theoretical Domains Framework; 2019. https://doi.org/10.1186/s13012-019-0884-4.

21. Staniford LJ, Schmidtke KA. A systematic review of hand-hygiene and environmental-disinfection interventions in settings with children. BMC Public Health. 2020;20(1):1-11. https://doi.org/10.1186/s12889-020-8301-0.

22. Spiteri K, Broom D, Bekhet AH, de Caro JX, Laventure B, Grafton K. Barriers and motivators of physical activity participation in middle-aged and older adults—a systematic review. J Aging Phys Act. 2019;27(6):929-44 https://doi. org/10.1123/japa.2018-0343.

\section{Publisher's Note}

Springer Nature remains neutral with regard to jurisdictional claims in published maps and institutional affiliations.
Ready to submit your research? Choose BMC and benefit from:

- fast, convenient online submission

- thorough peer review by experienced researchers in your field

- rapid publication on acceptance

- support for research data, including large and complex data types

- gold Open Access which fosters wider collaboration and increased citations

- maximum visibility for your research: over $100 \mathrm{M}$ website views per year

At BMC, research is always in progress.

Learn more biomedcentral.com/submissions 